\title{
Attentional selection of complex objects: Joint effects of surface uniformity and part structure
}

\author{
Lauren N. Hecht and Shaun P. Vecera \\ University of Iowa, Iowa City, Iowa
}

\begin{abstract}
What object properties warrant selection by object-based attention? Previous research has suggested that surface uniformity is required for object-based attentional selection (Watson \& Kramer, 1999), yet nonuniform objects are encountered frequently. In the present experiments, we investigated the interplay between surface uniformity and part boundaries and their effect on object-based attention. Specifically, we asked if attention can select nonuniform objects whose surface changes occur at part boundaries. Although uniformly colored objects did exhibit object-based effects, we only observed an object-based effect for multicolored objects when surface changes occurred at part boundaries. These findings suggest that attention can only select nonuniform objects when the surface change occurs at a part boundary.
\end{abstract}

Visual scenes are rich with information, but elementary visual information, such as local luminance values, do not appear to be perceived directly. Instead, elementary information is organized into perceptual groups (Palmer, 1999, 2002). Typical visual scenes will contain many perceptual groups, including objects and the regions that fall behind objects. To further manage the wealth of visual information in any scene, perceptual groups can guide attention in an object-based manner, allowing relevant objects to be selectively attended.

Several experimental designs have been used to examine object-based attention. Accuracy in feature reports (e.g., Duncan, 1984; Vecera \& Farah, 1994) and speeded spatial cuing tasks (e.g., Egly, Driver, \& Rafal, 1994; Vecera, 1994) are among the most common paradigms. For example, Egly et al. used a spatial cuing task in which observers viewed displays containing two simple objects, similar to those shown in Figure 1. Attention was summoned to a cued location at the end of one of two rectangles, and observers detected the onset of a target. Targets appeared at validly or invalidly cued locations; when targets were invalidly cued, they could appear in the cued object or in the uncued object. These two invalid locations were equidistant from the cued location, preventing any explanation of the results using location-based attention. Results from this task typically find that observers are fastest to respond to targets at the cued location and, more importantly, are faster to uncued targets in the cued object than those in the uncued object. This latter result defines an object-based attention effect.

Although there have been many demonstrations of object-based attention, few studies have attempted to determine the perceptual properties that define the objects selected by object-based attention. One relevant study by Watson and Kramer (1999) examined the perceptual grouping cues to which object-based attention was sensitive. Specifically, using a theory of perceptual organization proposed by Palmer and Rock (1994; Palmer, 1999, 2002), Watson and Kramer focused on the "uniform connectedness" cue, which states that uniform visual surfaces (i.e., surfaces with uniform color, luminance, or texture) are the foundation for perceptual organization. Object-based attention is also sensitive to uniform connectedness: An object-based effect was found for uniform objects that were composed of a single color and luminance but not for nonuniform objects that were composed of regions of different color and luminance. Thus, the objects selected by attention must have uniform surfaces, unless the nonuniform surfaces are task relevant; see Experiment 2 in Watson \& Kramer. This surface uniformity likely involves postconstancy reflectance of the surface (Rock, Nijhawan, Palmer, \& Tudor, 1992).

The finding that object-based attention typically selects only single-region objects may limit the generalizability of object-based attention because real world objects typically contain several regions (see Matsukura \& Vecera, 2006, for discussion; also see Martin, Fowlkes, \& Malik, 2004 for evidence from computer vision concerning image regions). Thus, Watson and Kramer's (1999) result likely represents a set of conditions under which surface uniformity is required for object-based attention, and other conditions might allow object-based attention to select nonuniform objects.

Various evidence supports the claim that object-based attention can select nonuniform objects under some conditions. Some of Watson and Kramer's (1999) own results indicated that making the multiple-regions of an object

L. N. Hecht, lauren-hecht@uiowa.edu or S.P.Vecera, shaun-vecera@uiowa.edu 


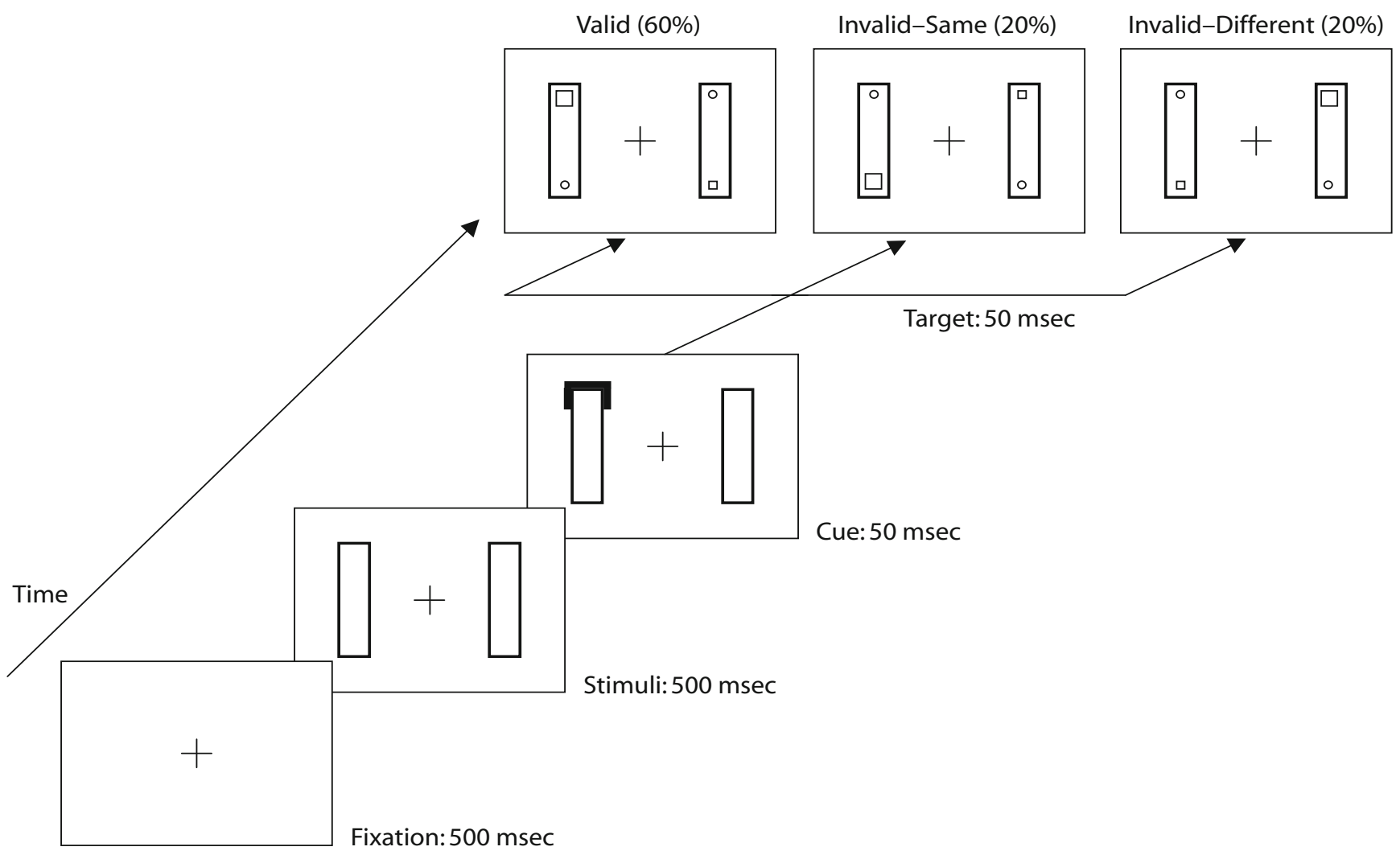

Figure 1. Event durations for a single trial in the spatial cuing task used in Experiments 1-5. Significantly faster responses on the invalid-same object trials than on the invalid-different object trials demonstrate an object-based effect of attention (see, e.g., Egly, Driver, \& Rafal, 1994). This effect has been replicated with black outline rectangles in a target identification task (see, e.g., Moore, Yantis, \& Vaughan, 1998; Pratt \& Sekuler, 2001).

task relevant would allow these objects to be selected by object-based attention. Additionally object-based effects are observed for occluded objects (Behrmann, Zemel, \& Mozer, 1998; Moore, Yantis, \& Vaughan, 1998) suggesting that separated (i.e., occluded) but uniform (i.e., similarly colored) regions can guide object-based attention. Further, Matsukura and Vecera (2006) recently reported that multiple-region objects could guide objectbased attention, provided that the different regions of an object were perceptually grouped. Thus, both top-down task relevance and bottom-up image grouping can allow multiple-region objects to be selected as a single unit by object-based attention.

The purpose of the present experiments is to further examine the perceptual information that allows multipleregion objects to guide object-based attention. Most real world objects contain multiple parts, and there are salient perceptual cues that occur at the boundaries between parts. Specifically, when two parts meet in an object, minima of curvature cues are created in the outline shape of the object (Hoffman \& Richards, 1984; Hoffman $\&$ Singh, 1997). We conjectured that there are regularities within objects in which surface changes (e.g., changes in luminance, color, or texture) tend to co-occur with part boundaries, as in the case of a lamp whose shade (e.g., white) is often a different color from its base (e.g., gold).
Our conjecture is consistent with Singh and Hoffman's (2001) part-coloring rule: Color changes occurring at part boundaries are interpreted as changes in surface reflectance. However, our work extends the part-coloring rule by examining effects of interactions between color changes and part boundaries on object-based attentional selection. Furthermore, our conjecture leads to a testable hypothesis for object-based selection of multiple-region objects: If surface changes occur more frequently across parts than within parts, then object-based attention should be guided by multiple-region objects in which surface changes occur at part boundaries. That is, we should see an object-based attention effect for multiple-region objects in which color changes occur at part boundaries, but not for objects in which color changes occur away from part boundaries.

The present experiments investigated part boundaries and surface uniformity in an object-based spatial cuing task (e.g., Egly et al., 1994; Vecera, 1994). We first replicated the presence of an object-based effect with uniform, single-part objects, followed by the absence of an objectbased effect with multiple-region, single-part objects. We then created multipart objects in which the surface of an outline object was composed of either one color or two different colors; for two-color objects, we manipulated whether the color border coincided with the part boundary or appeared off the part boundary. The color of the object's 
contours was considered a surface characteristic, consistent with previous research (Watson \& Kramer, 1999).

There were two possible outcomes from the latter experiments. If surface uniformity is a critical, strong constraint for object-based attention, then the object-based effect would not be present when there is any color dissimilarity, irrespective of the alignment of the surface colors and the part boundary. In contrast, if surface uniformity is modulated by part boundaries, then a significant objectbased effect would be present when color changes coincide with the part boundary but not when surface changes are misaligned with part boundaries.

To preview our results, Experiment 1 replicated objectbased attentional selection of uniform rectangles. Experiment 2 replicated the finding that nonuniform objects (i.e., objects made of different colors) do not guide object-based attention. Experiment 3 demonstrated that single-region, multipart objects guide object-based attention, consistent with previous findings (Vecera, Behrmann, \& McGoldrick, 2000; Vecera, Behrmann, \& Filapek, 2001). Experiments 4 and 5 demonstrated that object-based attention could select multiple-region objects when surface color changes occurred at part boundaries (Experiment 4), but not when they occurred away from part boundaries (Experiment 5).

\section{GENERAL METHOD}

\section{Participants}

Participants were University of Iowa undergraduates who volunteered for course credit and who reported having normal color vision and normal or corrected-to-normal acuity.

\section{Stimuli and Procedure}

Displays contained a white fixation cross subtending $0.4^{\circ}$ by $0.4^{\circ}$ of visual angle and two outline rectangles subtending $1.0^{\circ}$ wide by $6.0^{\circ}$ long of visual angle at a viewing distance of $70 \mathrm{~cm}$. The rectangles were aligned either horizontally or vertically, and the ends of each rectangle were equidistant from one another and fell $4.2^{\circ}$ from fixation. Stimuli were presented against a black background.

Observers' attention was cued to a location on one of the rectangles by brightening the edges at one end of a rectangle with a $0.1^{\circ}$ thick white line. The cue could appear at any of four ends of the rectangles. Following the cue, a target and three distractors-all white outline circles or squares, each occurring equally often - were presented at each of the four ends of the rectangles. The target stimuli were the large shapes measuring $0.9^{\circ}$ in diameter. The distractors were the small shapes subtending $0.5^{\circ}$ in diameter.

Targets appeared in three possible locations: the cued location (valid trials), the uncued end of the cued rectangle (invalid-same object trials), or the uncued rectangle (invalid-different object trials). The invalid target locations were equidistant from the cued location. Valid trials occurred on $60 \%$ of all trials, and the invalid conditions each occurred on $20 \%$ of all trials.

The event sequence is depicted in Figure 1. The fixation appeared for $500 \mathrm{msec}$, followed by the two rectangles. After the rectangles were present for $500 \mathrm{msec}$, a cue appeared for $50 \mathrm{msec}$. Immediately after the offset of the cue, one target and three distractors were presented for $50 \mathrm{msec}$. Observers pressed the " $n$ " key if the target was a large circle and the " $\mathrm{m}$ " key if the target was a large square. Observers received feedback after each response, followed by an intertrial interval of $500 \mathrm{msec}$. They received four blocks of 60 trials each and received a break between blocks. Each observer also received 24 unanalyzed practice trials.

\section{EXPERIMENT 1}

We first replicated the presence of an object-based effect for uniform, single-part objects.

\section{Method}

Participants. Twenty-one undergraduates participated in this experiment.

Stimuli. The contour of both rectangles was white (see Figure 2A).

\section{Results and Discussion}

One participant was excluded from the analyses because average accuracy dropped below $75 \%$ in at least one condition, resulting in few reaction times (RTs) in the invalid conditions. Mean RTs were calculated for correct trials that were slower than $150 \mathrm{msec}$ and faster than $2,000 \mathrm{msec}^{1}$ This trimming excluded less than $2 \%$ of trials.

Mean RTs and accuracy rates for the valid trials can be seen in Table 1 . The more theoretically relevant results from the invalid conditions are shown in Figure 3. An analysis of the three trial types (valid, invalid-same object, and invalid-different object) was significant $[F(2,19)=123.6$, $p<.05]$. Planned comparisons indicated that RTs on the valid trials $(556 \mathrm{msec})$ were significantly faster than those on the invalid-same $(661 \mathrm{msec})[t(19)=12.0, p<.05]$ and invalid-different object trials $(680 \mathrm{msec})[t(19)=13.8$, $p<.05]$. Critically, as shown in Figure 3, there was a significant difference between the invalid-same and invaliddifferent object trials $[t(19)=2.5, p<.05]$, indicating that object-based attention selected the uniform rectangles. Similar trends were found in the accuracy data, ruling out the possibility of a speed-accuracy tradeoff.

The present results replicated previous studies (e.g., Egly et al., 1994; Vecera, 1994) by demonstrating an object-based attention effect using our modified spa-

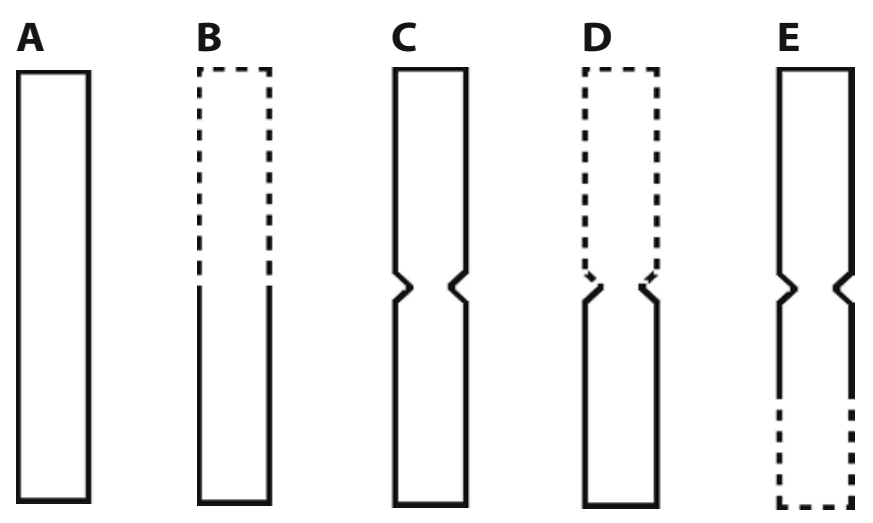

Figure 2. Sample stimuli used in Experiments 1-5 (A-E, respectively), with solid and dashed lines representing different color contours for multicolor displays (Experiments 2, 4, \& 5). Experiments 1 and 3 used a solid white contour. Displays containing one example of the display containing only the fixation and the objects from each experiment can be viewed on our laboratory's Web page at www.psychology.uiowa.edu/Faculty/Vecera/ lab/multipart.html. 
Table 1

Mean Reaction Times (RTs, in Milliseconds), Standard Errors (SEs), and Accuracy Rates for Valid Trials in Experiments 1-5

\begin{tabular}{cccc}
\hline & \multicolumn{2}{c}{$\mathrm{RT}$} & \\
\cline { 2 - 3 } Experiment & $M$ & $S E$ & \% Accuracy \\
\hline 1 & 556 & 17.1 & 97.12 \\
2 & 593 & 20.5 & 96.15 \\
3 & 574 & 15.4 & 96.65 \\
4 & 654 & 11.7 & 97.22 \\
5 & 626 & 22.0 & 95.35 \\
\hline
\end{tabular}

tial cuing paradigm. Having replicated this effect with single-region objects, we next addressed the treatment of multiple-region objects.

\section{EXPERIMENT 2}

We next replicated Watson and Kramer's (1999) absence of an object-based effect with multiple-region objects. Observers viewed displays containing two multiple-region objects, each containing two colors that met halfway through the object. Such objects should fail to produce an objectbased effect. Specifically, responses on invalid sameobject trials should be no faster than responses on invalid different-object trials.

\section{Method}

Participants. Twenty-two undergraduates participated in this experiment.

Stimuli. The contour of each rectangle was filled with two of four different colors. The RGB values for these colors are as follows: Red $=255,0,0$; green $=0,153,0$; yellow $=255,204,0$; purple $=$ $102,0,255$. The colored contour of each rectangle abruptly changed to the opposing color halfway through the object (see Figure 2B). The colors were completely intermixed and were presented ran- domly throughout the trials. Every color was present in each display and thus was not repeated within any given trial.

\section{Results and Discussion}

The criteria used in Experiment 1 were applied to the data from Experiment 2. The accuracy criterion excluded 2 participants from the analyses, and the RT trimming procedure excluded less than $2 \%$ of the data.

Again Table 1 shows RTs and accuracy rates for the valid trials, and Figure 3 presents the results from the invalid conditions. An analysis of the three trial types was significant $[F(2,19)=96.2, p<.05]$. Planned comparisons indicated that RTs on the valid trials $(593 \mathrm{msec})$ were significantly faster than those on the invalid-same $(710 \mathrm{msec})[t(19)=10.4, p<.05]$ and in the invaliddifferent object trials (702 msec) $[t(19)=12.1, p<.05]$. Critically, as shown in Figure 3, there was no significant difference between the invalid-same and invalid-different object trials $[t(19)=1.1$, n.s.], indicating that object-based attention did not select the nonuniform rectangles. The accuracy data mirrored the RT data, ruling out a speedaccuracy tradeoff.

The present results replicated previous studies demonstrating that object-based attention only selects objects with uniform surface features (Matsukura \& Vecera, 2006; Watson \& Kramer, 1999). Having replicated this nonuniformity effect, we next asked if the alignment of part structure and surface cues allows object-based attention to select nonuniform objects. Recall that there are two possible perspectives on the selection of multiple-region objects. On the one hand, regularities in real-world images suggest that changes in surface luminance or color may likely occur at part boundaries. Thus, when surface changes occur at a part boundary, the resulting perceptual object may be per-

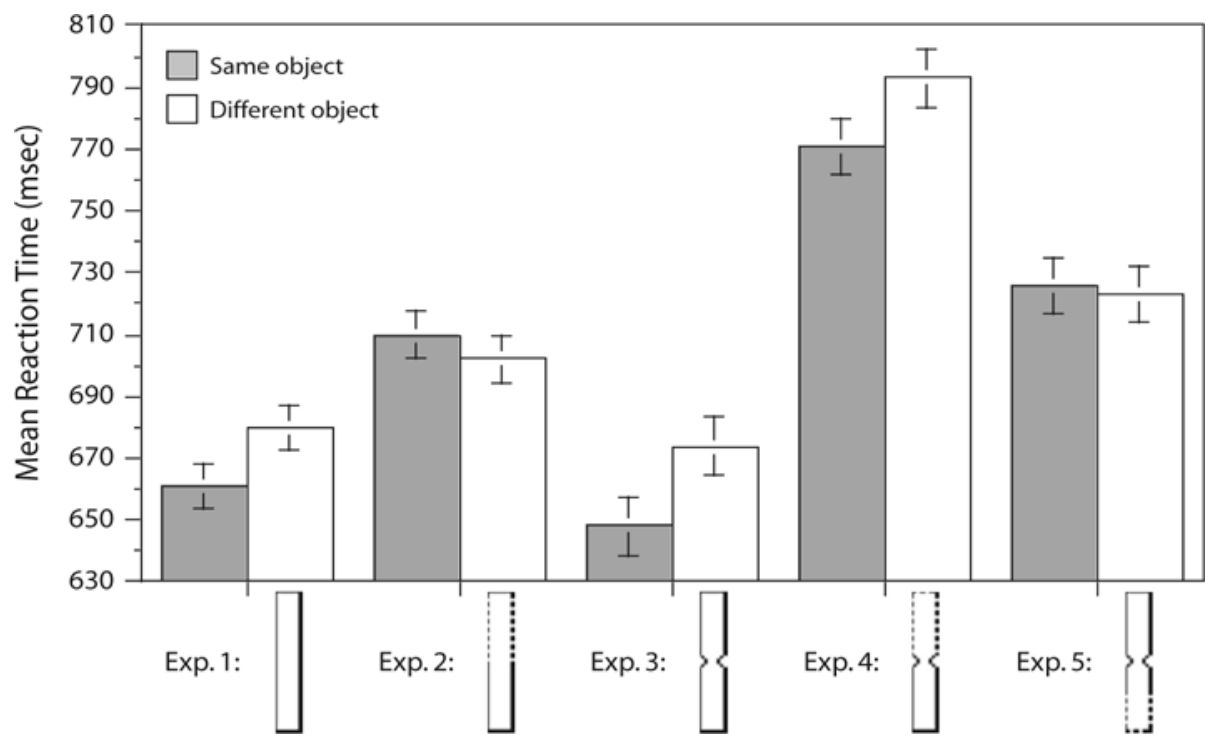

Figure 3. Mean reaction times on invalid-same and invalid-different object trials from Experiments 1-5. The numbers located on each column denote the accuracy for each condition. The error bars indicate $95 \%$ within-subjects confidence intervals computed on the same object versus different object comparisons for each experiment (Loftus \& Masson, 1994). 
ceived as a single multipart object to which attention can be directed, not perceived as the accidental alignment of two differently colored shapes. On the other hand, uniform connectedness might be a strong constraint on object perception, which cannot be easily modified by other perceptual information such as part boundaries. These possibilities were tested in Experiments 3-5.

\section{EXPERIMENT 3}

To address the potential interplay between surface uniformity and part boundaries, the remaining experiments used multipart objects and manipulated surface uniformity. We created these multipart objects by adding two minima of curvature points on the rectangles (see Figure $2 \mathrm{C}$ ). Before manipulating the surface uniformity of these multipart objects, we first ensured that these stimuli produced object-based effects.

\section{Method}

Participants. Twenty-one undergraduates participated in this experiment.

Stimuli. This experiment used multipart objects that contained minima of curvature at the middle of the rectangles, forming a salient part boundary. The minima of curvature were formed by a notch that was added to the length of the outline rectangles used in Experiment 1 (see Figure $2 \mathrm{C}$ ). This notch subtended $0.2^{\circ} \times 0.4^{\circ}$ of visual angle and was formed by a $90^{\circ}$ angle at the intersection of two lines that extended into the main body of the rectangle. To ensure objectbased attention could select these multipart objects, the objects were presented in a uniform color (white on a black background).

\section{Results and Discussion}

The data were analyzed as in Experiment 1. The accuracy criterion excluded one subject from the analyses, and the reaction time trimming procedure excluded less than $2 \%$ of the data.

Again Table 1 presents mean RTs and accuracy rates on the valid trials, and Figure 3 presents the results from the invalid trials. An analysis of the three trial type conditions was significant $[F(2,19)=61.3, p<.05]$. Planned comparisons indicated that RTs on the valid trials $(572 \mathrm{msec})$ were significantly faster than those on the invalid-same $(648 \mathrm{msec})[t(19)=7.2, p<.05]$ and the invalid-different $(674 \mathrm{msec})[t(19)=10.9, p<.05]$ object trials. Critically, there was a significant difference between the invalidsame and invalid-different object trials $[t(19)=2.8, p<$ $.05]$, demonstrating an object-based attention effect.

A similar trend was found in the accuracy for each trial type, including a trend toward higher accuracy on the invalidsame than invalid-different object trials. The present findings indicate that attention was able to select these multipart objects when they had a uniform surface, consistent with previous results that demonstrated object-based selection in multipart objects (e.g., Vecera et al., 2000, 2001).

\section{EXPERIMENT 4}

To directly test the relationship between surface uniformity and part structure, we created a surface nonuniformity that occurred at the part boundary of the multipart objects used in Experiment 3. If strict surface uniformity is required to engage object-based attention, then we should not observe an object-based effect, replicating the results of Experiment 2. In contrast, if object-based attention is engaged by surface changes occurring at part boundaries, then we should observe an object-based effect, replicating the results of Experiment 3.

\section{Method}

Participants. Twenty-four undergraduates participated in this experiment.

Stimuli. Each object contained two different colors, similar to the rectangles in Experiment 2. There were four colors total (two per object), and no color repeated within any trial. The colored contour of the objects changed abruptly halfway through the object at the part boundary, as shown in Figure 2D.

\section{Results and Discussion}

Three subjects' data were excluded based on the accuracy criterion used in the previous experiments, and another's data was excluded due to technical difficulties with the monitor. Less than $5 \%$ of the trials were excluded based on the RT trimming procedure.

Results from the valid trials appear in Table 1, and results from the invalid trials appear in Figure 3. An analysis of the three trial types was again significant $[F(2,19)=84.0, p<$ .05]. Planned comparisons indicated that RTs on the valid trials $(654 \mathrm{msec})$ were significantly faster than those on the invalid-same $(771 \mathrm{msec})[t(19)=10.3, p<.05]$ and invalid-different $(793 \mathrm{msec})[t(19)=10.1, p<.05]$ object trials. Critically, there was a significant difference between the invalid-same and invalid-different object trials $[t(19)=$ 2.53, $p<.05]$, showing a significant object-based effect. The accuracy data showed similar trends, indicating the absence of a speed-accuracy tradeoff.

The presence of the object-based effect suggests that attention selected the nonuniform objects when the surface change occurred at the part boundary. This finding supports our conjecture that the alignment of surface cues and part structure allows selection by object-based attention. Rather than adhering strictly to the cue of uniform connectedness, object-based attention may utilize statistical regularities that are present in real-world settings.

Although the present results suggest that aligning surface changes and part boundaries allows for effective object-based selection, there is one alternative explanation: Part structure along the object might attract objectbased attention more strongly than surface changes, allowing object-based attention to effectively ignore the surface nonuniformity. Thus, to conclusively argue that the alignment of surface changes and part boundaries is important for object-based attention, we must demonstrate that object-based effects can be eliminated in multipart objects in which surface changes are misaligned with part boundaries.

\section{EXPERIMENT 5}

In Experiment 5, we shifted the color change off the part boundary in our multipart objects. Now the color 
change occurred halfway through one part of the object, as shown in Figure 2E. If surface changes must occur at part boundaries in order to produce object-based attention effects, then the present manipulation should eliminate an object-based effect. In contrast, if multipart objects strongly attract object-based attention irrespective of surface uniformity, then the present manipulation should continue to produce an object-based attention effect.

\section{Method}

Participants. Twenty-one undergraduates participated in this experiment.

Stimuli. The stimuli were identical to those in Experiment 4, except now the multipart objects contained one color change occurring halfway through a single part of the object (see Figure 2E).

\section{Results and Discussion}

One subject was excluded from the analyses due to technical difficulties. No subjects were excluded based on the accuracy criterion, and the RT trimming procedure excluded less than $2 \%$ of trials.

Results from the valid trials appear in Table 1, and results from the invalid trials appear in Figure 3. An analysis of the three trial types was again significant $[F(2,19)=$ $22.1, p<.05]$, reflecting a validity effect. Planned comparisons indicated that RTs on the valid trials $(626 \mathrm{msec})$ were significantly faster than those on the invalid-same $(726 \mathrm{msec})[t(19)=5.0, p<.05]$ and the invalid-different $(723 \mathrm{msec})[t(19)=4.8, p<.05]$ object trials. Crucially, no difference was found between the invalid-same and invalid-different object trials $[t(19)=0.3$, n.s. $]$. The accuracy data showed similar trends. The absence of an objectbased effect suggests that object-based attention selects nonuniform, multipart objects only when the change occurs at the part boundary.

Finally, we compared our five experiments with a onefactor ANOVA on the object effects (invalid-different RTs minus invalid-same RTs). An analysis of the objectbased effects across the five experiments was significant $[F(4,95)=3.3, p<.05]$, indicating that the object-based effects were significantly different across the experiments. Planned comparisons indicated that object-based effects were larger in single-part uniform objects (Experiment 1) than in single-part nonuniform objects (Experiment 2) $[t(38)=2.5, p<.05]$. Most important, objectbased effects also were larger for multipart objects in which surface changes occurred at the part boundary (Experiment 4) than for multipart objects in which surface changes occurred off the part boundaries (Experiment 5) $[t(38)=2.0, p=.05]$.

Finally, there were no significant differences in the object-based effects for single- and multipart uniform objects (Experiments 1 and 3) $[t(38)<1$, n.s.], suggesting that a part boundary does not alter the size of object-based effects. Also, objects that contained a surface change at a part boundary (Experiment 4) showed object effects similar to those of uniform objects $[t(38)<1$, n.s.] for single-part objects (Experiment 1 ) and $[t(38)<1$, n.s. $]$ for multipart objects (Experiment 3$){ }^{2}$

\section{GENERAL DISCUSSION}

Our experiments demonstrated that surface uniformity does not appear to be a mandatory characteristic of the objects that object-based attention selects. Instead, surface nonuniformities are acceptable to object-based selection processes if they occur at a part boundary. Specifically, when an object undergoes a surface color change at the part boundary, object-based attention selects the object, producing an object-based attention effect. However, when a surface change occurs at no part boundary (Experiment 2) or occurs off of the part boundary (Experiment 5), objects are not readily selected by object-based attention and no object-based effects are observed.

The present findings suggest that uniform surface characteristics - uniform connectedness - are not a penultimate constraint on object-based attention. Although uniform connectedness is an important cue that affects both perceptual organization (Palmer \& Rock, 1994) and object-based attention (Watson \& Kramer, 1999), other cues, such as part boundaries in the present experiments, can affect the use of uniform connectedness. Indeed, in a previous study, Matsukura and Vecera (2006) demonstrated that nonuniform objects could be selected by object-based attention if the different regions were grouped together by closure and common region cues. In these studies, object-based effects were present for nonuniform objects when the outer edge of the objects was enclosed by a uniform, closed contour. The present experiments extend Matsukura and Vecera's results by demonstrating a modulating effect on uniform connectedness with more ecologically valid stimuli. Other ecologically valid changes to objects, such as the presence of cast shadows that create nonuniformities in surface brightness across objects, may allow object-based attention to override uniform connectedness and select such objects.

The surface properties of some objects do not adhere to our claim that surface changes tend to occur at part boundaries. For example, the stripes on a tiger or spots on a cow violate our conjecture. However, in the case of animals, which are most likely object category to violate our conjecture, the misalignment of part boundaries and surface changes might be characteristic of camouflage patterns. Camouflage could disrupt object-based attentional selection and increase the difficulty of recognizing camouflaged objects. Although camouflage can be broken and objects recognized, such recognition might be based on other cues in real world scenes (e.g., movement) or based on familiarity of the object.

Our results are consistent with the conjecture that surface changes are likely to occur at part boundaries in real world objects. Of course, we have not directly tested this in the present experiments, but the influence of object regularities on object-based attention makes testable predictions that could be addressed by examining real-world images. There is a growing literature that seeks to explain many perceptual phenomena through image-based statistical regularities (e.g., Howe \& Purves, 2004, 2005; Geisler, Perry, Super, \& Gallogly, 2001), and studies of object-based attention could benefit from similar analyses 
by linking object-based attention to the perceptual information important for forming objects out of the raw sensory data that is reflected into the eye.

\section{AUTHOR NOTE}

This research was supported by NSF Grant BCS 03-39171 awarded to S.P.V. We thank Joshua Cosman and Benjamin Lester for assisting in data collection. We also extend our thanks to Robert Nosofsky and three anonymous reviewers for their feedback on an earlier revision of this article. Correspondence concerning this article should be addressed to L. N Hecht or S. P. Vecera, Department of Psychology, 11 Seashore Hall E, University of Iowa, Iowa City, IA 52242-1407 (e-mail: lauren-hecht @uiowa.edu or shaun-vecera@uiowa.edu).

\section{REFERENCES}

Behrmann, M., Zemel, R. S., \& Mozer, M. C. (1998). Object-based attention and occlusion: Evidence from normal participants and a computational model. Journal of Experimental Psychology: Human Perception \& Performance, 24, 1011-1036.

Duncan, J. (1984). Selective attention and the organization of visual information. Journal of Experimental Psychology: General, 113, 501-517.

Egly, R., Driver, J., \& Rafal, R. D. (1994). Shifting visual attention between objects and locations: Evidence from normal and parietal lesion subjects. Journal of Experimental Psychology: General, 123, 161-177.

Geisler, W. S., Perry, J. S., Super, B. J., \& Gallogly, D. P. (2001). Edge co-occurrence in natural images predicts contour grouping performance. Vision Research, 41, 711-724.

Hoffman, D. D., \& Richards, W. A. (1984). Parts of recognition. Cognition, 18, 65-96.

Hoffman, D. D., \& Singh, M. (1997). Salience of visual parts. Cognition, 63, 29-78.

Howe, C. Q., \& Purves, D. (2004). Size contrast and assimilation explained by the statistics of natural scene geometry. Journal of Cognitive Neuroscience, 16, 90-102.

Howe, C. Q., \& Purves, D. (2005). Natural scene geometry predicts the perception of angles and line orientation. Proceedings of the National Academy of Sciences, 102, 1228-1233.

Loftus, G. R., \& Masson, M. E. J. (1994). Using confidence intervals in within-subjects designs. Psychonomic Bulletin \& Review, 1, 476-490.

Martin, D., Fowlkes, C., \& MaliK, J. (2004). Learning to detect natural image boundaries using local brightness, color and texture cues. IEEE Transactions on Pattern Analysis \& Machine Intelligence, 26, 530-549.

Matsukura, M., \& Vecera, S. P. (2006). The return of object-based attention: Selection of multiple-region objects. Perception \& Psychophysics, 68, 1163-1175.

Moore, C. M., Yantis, S., \& Vaughan, B. (1998). Object-based visual selection: Evidence from perceptual completion. Psychological Science, 9, 104-110.

Palmer, S. E. (1999). Vision science: Photons to phenomenology. Cambridge, MA: MIT Press.

Palmer, S. E. (2002). Perceptual organization in vision. In H. [E.] Pashler \& S. Yantis (Eds.), Stevens 'handbook of experimental psychology:
Vol. 1. Sensation and perception (3rd ed., pp. 177-234). Hoboken, NJ: Wiley.

PAlmer, S., \& Rock, I. (1994). Rethinking perceptual organization: The role of uniform connectedness. Psychonomic Bulletin \& Review, 1, 29-55.

Pratt, J., \& Sekuler, A. B. (2001). The effects of occlusion and past experience on the allocation of object-based attention. Psychonomic Bulletin \& Review, 8, 721-727.

RATCLIFF, R. (1993). Methods for dealing with reaction time outliers. Psychological Bulletin, 114, 510-532.

Rock, I., Nijhawan, R., Palmer, S., \& Tudor, L. (1992). Grouping based on phenomenal similarity of achromatic color. Perception, 21, 779-789.

Singh, M., \& Hoffman, D. D. (2001). Part-based representations of visual shape and implications for visual cognition. In T. F. Shipley \& P. J. Kellman (Eds.), From fragments to objects: Segmentation and grouping in vision (pp. 401-459). New York: Elsevier.

VeCERA, S. P. (1994). Grouped locations and object-based attention: Comment on Egly, Driver, \& Rafal (1994). Journal of Experimental Psychology: General, 123, 316-320.

Vecera, S. P., Behrmann, M., \& Filapek, J. C. (2001). Attending to the parts of a single object: Part-based selection limitations. Perception \& Psychophysics, 63, 308-321.

Vecera, S. P., Behrmann, M., \& McGoldrick, J. (2000). Selective attention to the parts of an object. Psychonomic Bulletin \& Review, 7, 301-308.

Vecera, S. P., \& Farah, M. J. (1994). Does visual attention select objects or locations? Journal of Experimental Psychology: General, 123, 146-160.

Vecera, S. P., Vogel, E. K., \& Woodman, G. F. (2002). Lower region: A new cue for figure-ground assignment. Journal of Experimental Psychology: General, 131, 194-205.

Watson, S. E., \& Kramer, A. F. (1999). Object-based visual selective attention and perceptual organization. Perception \& Psychophysics, 61, 31-49.

\section{NOTES}

1. Following suggestions from Ratcliff (1993), we examined our data using different response time analyses (e.g., median, trimmed means, etc.) The pattern of results in all of our experiments remains similar across different response time analyses.

2. Although the use of different subjects in each experiment is likely to account for baseline reaction time differences between experiments, there is an alternative explanation for the overall lengthening of reaction times in Experiment 4. Similar increases in baseline reaction time have been observed in conditions containing multiple constraints on the interpretation of an image. For example, Vecera, Vogel, \& Woodman (2002) found figure-ground displays containing a strong cue for figure-ground assignment slowed reaction times compared to displays lacking figure-ground cues. They proposed that additional processing time was required because the figure-ground cue constrains the interpretation of the image, increasing the reaction times overall. We would speculate that the same happens when surface cues and part cues align: The alignment provides a constraint on image interpretation that is absent when the cues are misaligned or absent, so baseline reaction times differ across these conditions.

(Manuscript received May 11, 2006; revision accepted for publication January 26, 2007.) 